\title{
A Longitudinal Study on the Mental Health of College Students in Jinan During the Peak Stage of the COVID-19 Epidemic and the Society Reopening
}

\author{
Xiaolei Zhenga,b Yuji Guo ${ }^{c}$ Wen Ma $^{d}$ Hui Yanga Liyan Luo ${ }^{\mathrm{e}}$ Li Wen $^{f}$ \\ Xiaolan Khou $^{d}$ Qing $\mathrm{Li}^{\mathrm{a}}$ Jianzhong $\mathrm{Bi}^{\mathrm{a}}$ Ping Wang ${ }^{\mathrm{a}}$ Hongxing Wang ${ }^{\mathrm{b}, \mathrm{g}, \mathrm{h}}$ \\ aDepartment of Neurology, The Second Hospital, Cheeloo College of Medicine, Shandong University, Jinan, China; \\ ${ }^{b}$ Division of Neuropsychiatry and Psychosomatics, Department of Neurology, Xuanwu Hospital, Capital Medical \\ University, Beijing, China; 'Department of Histology and Embryology, Cheeloo College of Medicine, Shandong \\ University, Jinan, China; ${ }^{d}$ Center for Clinical Neurolinguistics, School of Foreign Languages and Literature, \\ Shandong University, Jinan, China; 'Department of Epidemiology, School of Public Health, Cheeloo College of

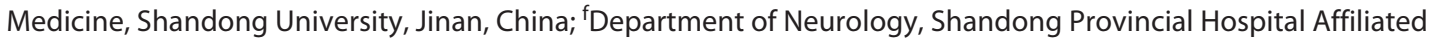 \\ to Shandong First Medical University, Jinan, China; 9 Beijing Key Laboratory of Neuromodulation, Beijing, China; \\ hBeijing Psychosomatic Disease Consultation Center, Xuanwu Hospital, Capital Medical University, Beijing, China
}

\section{Keywords}

Coronavirus disease $2019 \cdot$ Mental health · College students · Depression · Anxiety · Insomnia

\begin{abstract}
Introduction: COVID-19, a continuously emerging humanto-human infectious disease, has exerted a significant impact on the mental health of college students. However, little is known regarding the variations in the mental health issues experienced by college students during the peak versus reopening stages of the COVID-19 epidemic in China. Methods: To assess these issues, an online longitudinal survey was conducted via a WeChat applet. Undergraduates $(n=$ 300) were recruited from 26 universities throughout Jinan in February 2020 (T1 - the epidemic peak stage) and in January 2021 ( $T 2$ - the society reopening stage). Their mental status was determined using the Patient Health Questionnaire-9, the Generalized Anxiety Disorder-7 item, and the Insomnia Severity Index. Results: Of the original 300 college students
\end{abstract}

karger@karger.com www.karger.com/bmh

Karger $\stackrel{\text { ' }}{5}$

GOPEN ACCESS
(C) 2021 The Author(s)

Published by S. Karger AG, Basel

This is an Open Access article licensed under the Creative Commons Attribution-NonCommercial-4.0 International License (CC BY-NC) (http://www.karger.com/Services/OpenAccessLicense), applicable to the online version of the article only. Usage and distribution for commercial purposes requires written permission. recruited for this survey, 294 responses at T1 and 285 at T2 were analyzed. Compared with responses obtained at T1, college students at $\mathrm{T} 2$ showed a greater prevalence of depression ( 65.3 vs. $51.0 \% ; p=0.001)$ and anxiety (47.7 vs. $38.1 \%, p=0.019)$, and experienced more severe depression $(p<0.001)$ and anxiety $(p<0.001)$. Both males $(p=0.03)$ and females $(p<0.01)$ showed higher levels of depression at T2 versus $\mathrm{T} 1$, while no differences were obtained with regard to anxiety and insomnia. At T1, Grade 4 students showed greater levels of depression $(p=0.005)$ and anxiety $(p=0.008)$ than that of Grade 1 students. While at T2, only greater levels of depression $(p=0.004)$ were present when compared with that of Grade 1 students. Additionally, Grade 4 college students demonstrated a greater prevalence of depression at $\mathrm{T} 2$ versus $\mathrm{T} 1(p=0.03)$, but no statistically differences were present for anxiety and insomnia. No statistically significant differences were obtained among the 4 grades of college students for insomnia at either the T1 or T2. Conclusion: With progression of the COVID-19 epidemic, college students showed increasing levels of depression and anxiety,
Correspondence to:

Ping Wang, wping0108@163.com

Hongxing Wang, wanghongxing@xwh.ccmu.edu.cn 
with Grade 4 college students being most seriously affected. It is imperative that intervention strategies be implemented to mitigate against these mental health issues resulting from the COVID-19 epidemic.

(C) 2021 The Author(s).

Published by S. Karger AG, Basel

\section{Introduction}

The World Health Organization (WHO) declared that the COVID-19 outbreak was an ongoing global health emergency [1,2]. Globally, as of May 31, 2021, there were $3,016,005$ confirmed cases of COVID-19, including 73,807 deaths, as reported to the WHO [3]. Of continued concern, this epidemic is still raging worldwide [4]. At its maximal level of infection, COVID-19 represented a lifethreatening condition and was reported to induce various psychological problems within the general population [5], medical personnel [6], and college students [7, 8]. Moreover, other factors related to COVID-19 such as changes in daily life activities (working and studying at home), social distancing, and economic recession further affected the mental health of Chinese people [9].

After the outbreak of COVID-19 in China, daily routines of college students were markedly disrupted, due to adjustments required for online learning platforms and maintenance of social distancing. In these Chinese college students, their psychological problems were reported during the peak stage of the COVID-19 epidemic [7]. According to findings from a previous study, psychological problems are the main obstacle to academic success in college students [10]. Psychological problems can interfere with concentration, motivation, and social interactions, all of which are considered as key factors for the success of student's academic performance [11]. As based on results of studies involving severe acute respiratory syndrome, the outbreak of an infectious disease exerts considerable distress upon people who had been quarantined, some of whom continued to experience psychological issues persisting for 6 or even 36 months after release from quarantine $[12,13]$. Therefore, from the peak stage of the COVID-19 epidemic to the reopening of society, and even into the post-COVID-19 era, the mental health status and psychological problems of college students require long-term attention.

According to the timeline of the COVID-19 epidemic, China achieved peak infection rates in February 2020 and reopened to society at the end of April 2020. Chinese college students then returned to campus in mid-May 2020 [14]. An assessment of the mental health and psycholog- ical problems of these students were performed during the peak stage of the COVID-19 epidemic in China [7, 15]. However, few reports on the mental health of these college students, in particular, a lack of prospective longitudinal research, have been conducted during the reopening stage of this epidemic. Given the changes in their daily learning environment (from campus to home) as experienced from the peak to the reopening stage of the COVID-19 epidemic, the mental status of college students may have varied markedly. Accordingly, we proposed that college students within the universities of Jinan may have encountered a greater prevalence and more severe anxiety, depression, and insomnia during peak stage versus the reopening as reported by self-ratings. To assess this possibility, in this study, we collected data from college students at universities in Jinan City, and prospectively investigated the prevalence and the severities of anxiety, depression, and insomnia as experienced during the peak (T1) and reopening (T2) stages of the COVID-19 epidemic.

\section{Materials and Methods}

\section{Participants}

A total of 300 Chinese undergraduate students (age $\geq 18$ years) at universities within Jinan City were recruited for participation in this online survey. Subjects were excluded if they had self-reported current or lifetime mental disorders, substance abuse, or current physical diseases including COVID-19 infection. The research was approved by the Medical Ethics Committee of the Second Hospital of Shandong University (No. KYLL-2020 [LW]-062). All procedures contributing to this work comply with the ethical standards of relevant national and institutional committees on human experimentation and are in accord with the Helsinki Declaration of 1975 , as revised in 2008 .

\section{Design and Procedure}

A longitudinal study was conducted via an online survey. The first survey was conducted over the period from February 19-29 of 2020 (T1), which represented the peak stage of the COVID-19 outbreak in China [16]. T1 then included the quarantine period when college students were away from campus and therefore required that they adapt to online study and social distancing. The second survey was administered over the period from January 1529 of 2021 (T2), that is, 12 months after the COVID-19 outbreak in China. This T2 period then corresponded with the reopening stage of society when Chinese students gradually returned to their "normal" campus life [17].

Participants were recruited via WeChat groups from 26 universities within Jinan. Those who agreed to participate in the study were informed of the purpose of the survey, that is, to better understand the mental status of college students during and after the COVID-19 epidemic. Students completed the surveys using a questionnaire via a WeChat applet at T1 and T2. In order to certify the quality and completeness of the questionnaire, a simple 
math question (i.e., 21-5 = ?) was included at the end of the survey. Participants failing to complete the survey received a notice about pending questions from the online applet when submitting the questionnaire. The online applet did not present such warnings to those who gave up.

\section{Measurements}

Demographic variables including age, gender (male and female), and grade (i.e., Grade 1, Grade 2, Grade 3, and Grade 4) were collected via a questionnaire. Participants were also requested whether they had a history of physical diseases, or mental illnesses before COVID-19 and whether they were having physical diseases (the question was "Do you currently have any physical disease including? [diagnosed by medical examination in the hospital]"), those who answered positively were excluded. Additionally, insomnia, anxiety, and depression symptoms were evaluated.

The Patient Health Questionnaire (PHQ-9) was applied to examine depression symptoms [18]. It is a 9-item self-report scale, and its items pertain to the Diagnostic and Statistical Manual of Mental Disorders criteria for major depressive disorder [19]. The items are scored on a 4-point scale ranging from 0 to 3 ( 0 point for not at all, 1 point for several days, 2 points for more than half of the days, and 3 points for nearly every day). And participants rate the items according their situation during the past 2 weeks. Previous studies suggest that the total score ranges from 0 to $27(0-4$ : without depression symptoms, 5-9: with mild depression symptoms, 10-14: with moderate depression symptoms, 15-19: with moderate to severe depression symptoms, and 20-27: with severe depression symptoms) [20]. Previous studies revealed that the scale of the PHQ-9 showed good internal consistency, in which the reliability and validity of the Chinese version have been demonstrated [21].

The 7-item Generalized Anxiety Disorder Scale (GAD-7) was the most widely used to detect the symptoms of anxiety and has been proven to be a valid and efficient tool for screening for GAD and assessing its severity [22]. It is a 7-item self-report scale, and the items are scored on a 4-point scale ranging from 0 to 3 ( 0 point for not at all, 1 point for several days, 2 points for more than half of the days, and 3 points for nearly every day). Participants rate the items according their situation during the preceding 2 weeks. The score was scaled from 0 to 21 (0-4: without anxiety symptoms, 5-9: with mild anxiety symptoms, 10-13: with moderate anxiety symptoms, 14-18: with moderate to severe anxiety symptoms, and 19-21: with severe anxiety symptoms) [23]. The reliability and validity of the Chinese version of the GAD-7 have been presented [7].

The Insomnia Severity Index was a reliable and valid instrument to quantify perceived insomnia severity, which is likely to be a clinically useful tool as a screening device or as an outcome measure in insomnia treatment research. It is composed of 7 items that evaluate the following situations: the severity of initial, middle, and late insomnia, sleep problem interference with daily functioning, satisfaction with current sleep pattern, noticeability of impairment caused by the sleep problem, and worry about sleep problems [24]. The items are scored on a 5-point scale ranging from 0 to 4 . Participants rate the items according their situation during the preceding 2 weeks. The score was scaled from 0 to 28 (0-7: without insomnia, 8-14: with mild insomnia, 15-21: with moderate insomnia, and 22-28: with severe insomnia).

\section{Statistical Analysis}

All statistical analyses were performed with use of the IBM SPSS Statistical Software program (version 19). In the current study, numerical data were presented as counts (percentage of the total), and $\chi^{2}$ tests were used to compare group differences of categorical variables, while Mann-Whitney tests were used to compare independent groups on continuous variables that were nonnormally distributed. Descriptive statistics included mean values and standard deviations for continuous and non-normally distributed data, with the ANOVA used to assess group differences. Bonferroni's post hoc multiple comparison tests were used to assess pairwise differences among groups. A $p$ value of $<0.05$ was required for results to be considered as statistically significant.

\section{Results}

All 300 college students completed the questionnaire at T1 (Grade 1: $n=84,28.0 \%$; Grade 2: $n=100,33.3 \%$; Grade 3: $n=65,21.7 \%$; and Grade 4: $n=51,17.0 \%)$. Among these 300 students, 6 were excluded ( 2 had previous mental disorders and 4 had organic diseases) resulting in a final sample of 294 for analyses. At T2, 15 subjects were not included in the analysis ( 9 did not respond to the survey, 2 had previous mental disorders and 4 had organic diseases). Therefore, 291 subjects completed the questionnaire (Grade 1: $n=81,27.8 \%$; Grade 2: $n=100$, 34.4\%; Grade 3: $n=63,21.6 \%$; and Grade $4: n=47,16.2 \%$ ) and 285 were analyzed. As shown in online supplementary Table 1 (for all online supplementary material, see www.karger.com/doi/10.1159/000519586) no differences with regard to age, gender, and grade of participants between the T1 and T2 assessments were present.

As shown in Table 1, compared with those at T1, college students at T2 showed greater prevalence rates of depression (65.3 vs. $51.0 \% ; p=0.001$ ) and anxiety (47.7 vs. $38.1 \%, p=0.019)$. Moreover, college students at T2 experienced more severe levels of depression $(p<0.001)$ and anxiety $(p<0.001)$ than those at T1. No significant differences in the prevalence or severity of insomnia were observed among college students between T1 and T2.

When evaluating differences in levels of depression, anxiety, and insomnia in college students as based on gender at T1 versus T2, we found that both males ( $p=$ $0.03)$ and females $(p<0.01)$ reported higher levels of depression at T2 than at T1, but no differences were found for anxiety and insomnia (Fig. 1). At T1, there was a marginally significant difference in the prevalence of depression in females versus males (55.1 vs. $42.7 \%$; $p=0.047$ ), but no significant differences in anxiety and insomnia between these 2 groups (Table 2). At T2, no statistically significant differences were present between females and 
Table 1. Mental health status of the college students in February 2020 (T1) and January 2021 (T2)

\begin{tabular}{|c|c|c|c|}
\hline Characteristics & $\begin{array}{l}\text { T1 participants } \\
(n=294)\end{array}$ & $\begin{array}{l}\text { T2 participants } \\
(n=285)\end{array}$ & $p$ value \\
\hline \multicolumn{4}{|l|}{ PHQ-9 (depression) } \\
\hline 0-4 (asymptomatic) & $144(49.0)$ & $99(34.7)$ & \multirow{2}{*}{0.001} \\
\hline 5-27 (symptomatic) & $150(51.0)$ & $186(65.3)$ & \\
\hline \multicolumn{4}{|l|}{ Degree of depression by PHQ-9 } \\
\hline $5-9$ (mild) & $88(29.9)$ & $81(28.4)$ & \multirow{4}{*}{$<0.001$} \\
\hline 10-14 (moderate) & $42(14.3)$ & $61(21.4)$ & \\
\hline 15-19 (moderate to severe) & $13(4.4)$ & $34(12.0)$ & \\
\hline 20-27 (severe) & $7(2.4)$ & $10(3.5)$ & \\
\hline \multicolumn{4}{|l|}{ GAD-7 (anxiety) } \\
\hline 0-4 (asymptomatic) & $182(61.9)$ & $149(52.3)$ & \multirow{2}{*}{0.019} \\
\hline 5-21 (symptomatic) & $112(38.1)$ & $136(47.7)$ & \\
\hline \multicolumn{4}{|l|}{ Degree of anxiety by GAD-7 } \\
\hline $5-9$ (mild) & $98(33.3)$ & $80(28.0)$ & \multirow{4}{*}{$<0.001$} \\
\hline 10-13 (moderate) & $7(2.4)$ & $29(10.2)$ & \\
\hline 14-18 (moderate to severe) & $5(1.7)$ & $18(6.3)$ & \\
\hline 19-21 (severe) & $2(0.7)$ & $9(3.2)$ & \\
\hline \multicolumn{4}{|l|}{ ISI (insomnia) } \\
\hline 0-7 (asymptomatic) & $197(67.0)$ & $192(67.4)$ & \multirow{2}{*}{0.926} \\
\hline 8-28 (symptomatic) & $97(33.0)$ & $93(32.6)$ & \\
\hline \multicolumn{4}{|l|}{ Degree of insomnia by ISI } \\
\hline 8-14 (mild) & 79 (26.9) & $73(25.6)$ & \multirow{3}{*}{0.966} \\
\hline 15-21 (moderate) & $11(3.7)$ & $18(6.3)$ & \\
\hline $22-28$ (severe) & $7(2.4)$ & $2(0.7)$ & \\
\hline
\end{tabular}

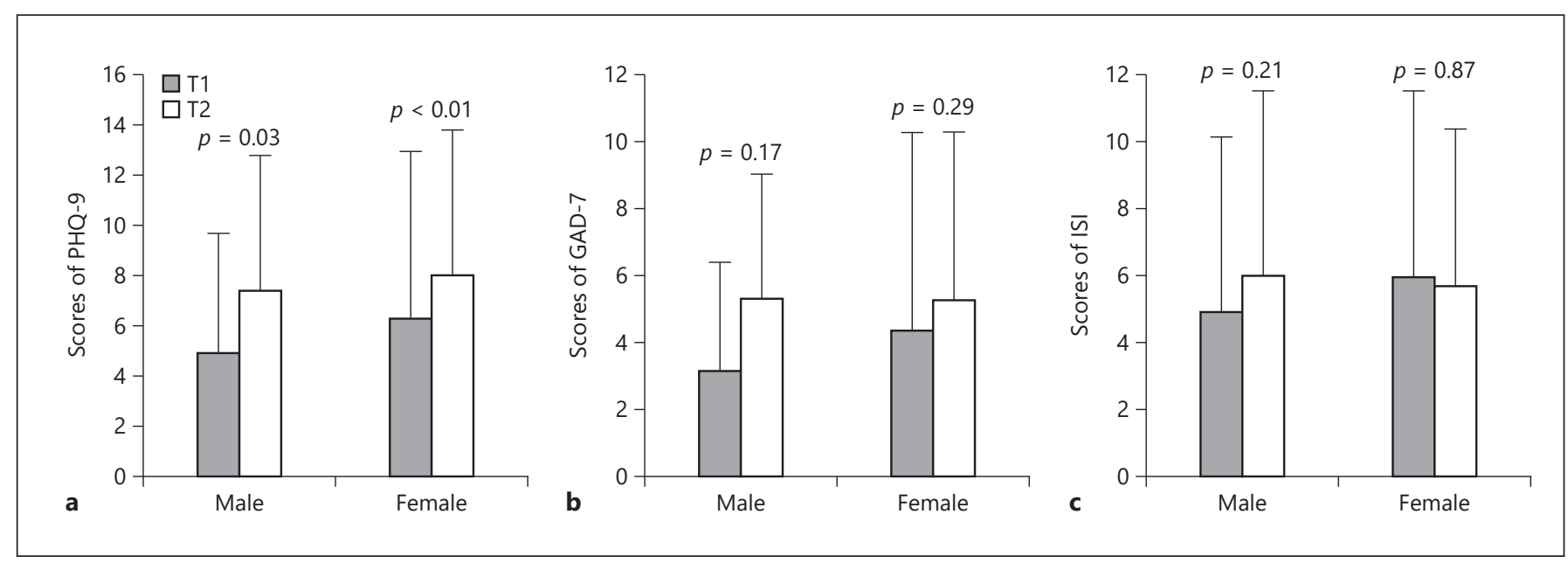

Fig. 1. Levels of depression, anxiety, and insomnia in males and females at February 2020 (T1) and January 2021 (T2). Depression levels (PHQ-9, range of scores: 0-27) (a); anxiety levels (GAD-7, range of scores: 0-21) (b); insomnia levels (ISI, range of scores: 0-28) (c). PHQ-9, Patient Health Questionnaire-9; GAD-7, Generalized Anxiety Disorder-7 item; ISI, Insomnia Severity Index. 
Table 2. Prevalence of depression, anxiety, and insomnia in males and females at T1 and T2

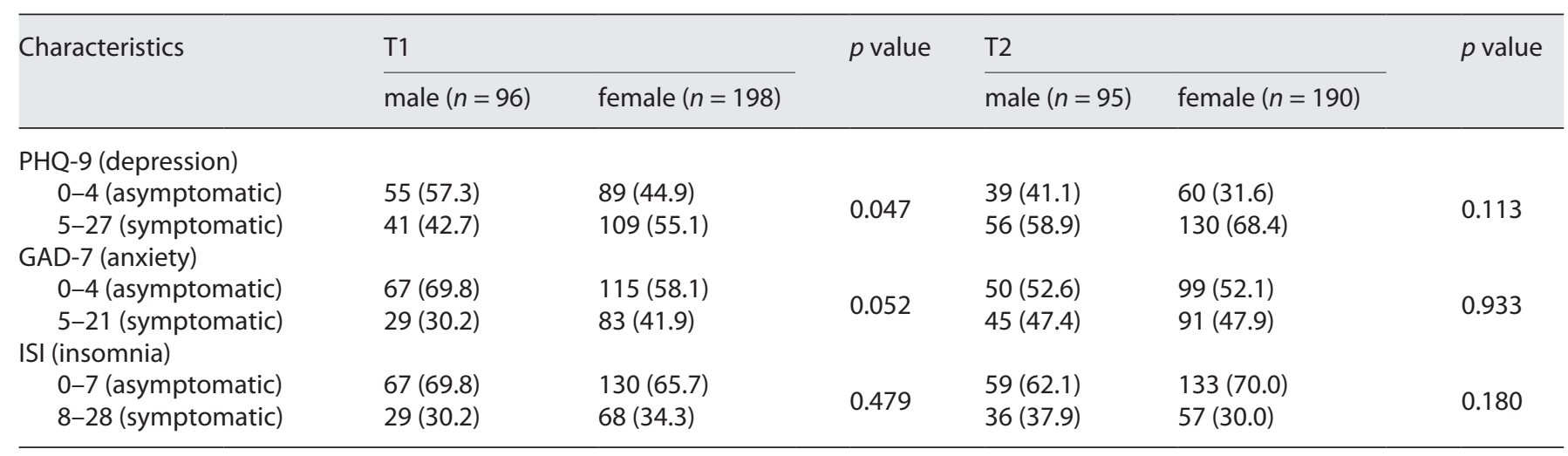

Values are presented as $n(\%) ; x^{2}$ tests were used for analyses of group differences of categorical variables. T1, February 2020; T2, January 2021; PHQ-9, Patient Health Questionnaire-9; GAD-7, Generalized Anxiety Disorder-7 item; ISI, Insomnia Severity Index.
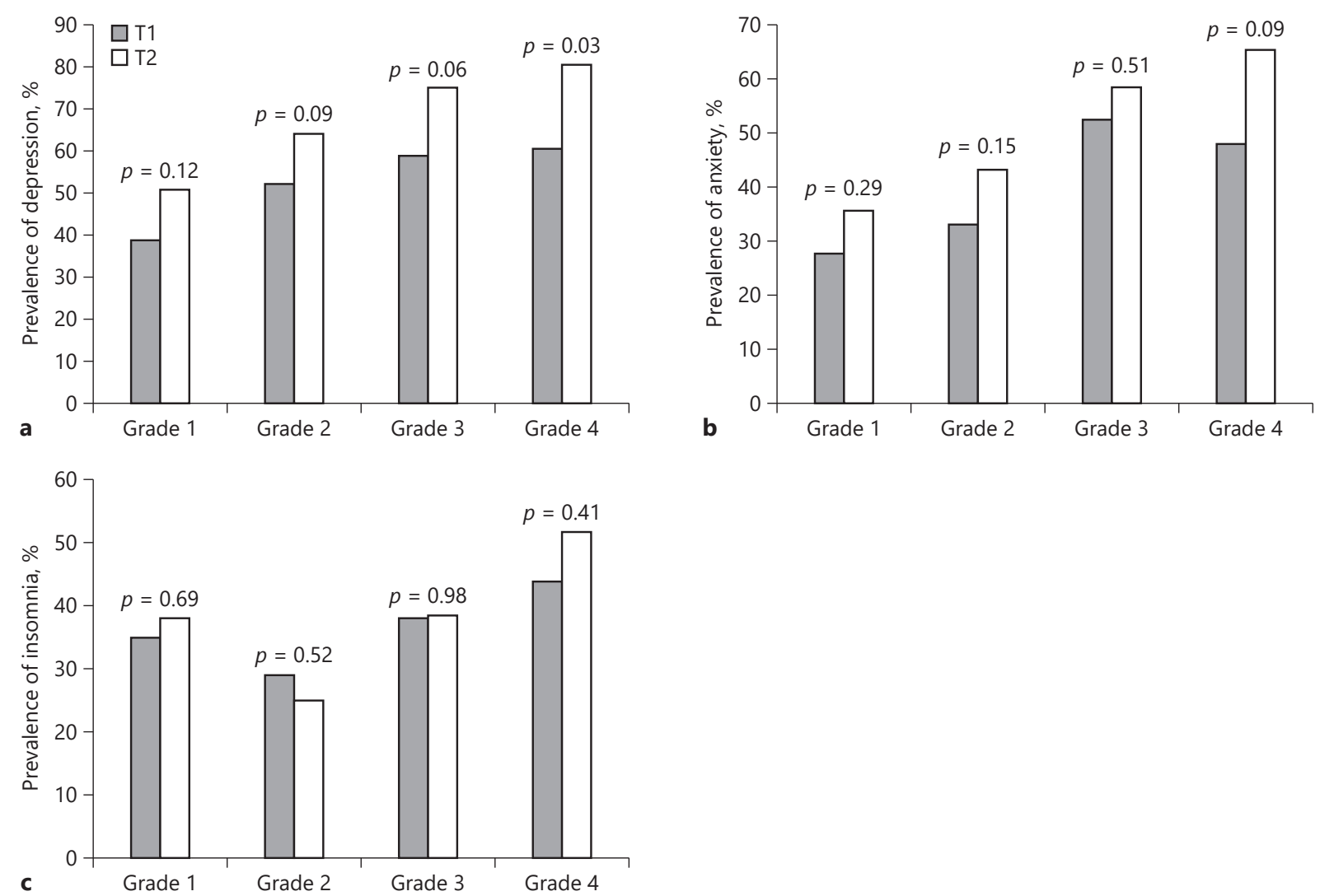

Fig. 2. Prevalence of depression, anxiety, and insomnia among the different grades of college students in February 2020 (T1) and January 2021 (T2). Depression (a); anxiety (b); insomnia (c). PHQ-9, Patient Health Questionnaire-9; GAD-7, Generalized Anxiety Disorder-7 item; ISI, Insomnia Severity Index. 
Table 3. Depression, anxiety, and insomnia among different grades of college students at T1 and T2

\begin{tabular}{lllllll}
\hline Characteristics & Total & Grade 1 & Grade 2 & Grade 3 & Grade 4 & $p$ value \\
\hline T1 & & & & & & \\
Depression (PHQ-9, Range: 0-27) & $5.92(5.25)$ & $4.73(5.09)$ & $5.77(4.78)$ & $7.00(5.76)$ & $8.14(5.74)$ & 0.007 \\
$\quad \begin{array}{l}\text { Anxiety (GAD-7, Range: 0-21) } \\
\text { Insomnia (ISI, Range: 0-28) }\end{array}$ & $4.13(3.71)$ & $3.34(3.95)$ & $3.75(2.84)$ & $5.61(4.13)$ & $5.71(4.16)$ & 0.001 \\
& $5.66(5.50)$ & $4.83(5.43)$ & $5.65(5.62)$ & $6.15(4.84)$ & $7.17(5.79)$ & 0.188 \\
T2 & & & & & & \\
$\quad$ Depression (PHQ-9, Range: 0-27) & $8.03(6.11)$ & $5.66(5.62)$ & $7.19(5.80)$ & $9.60(6.37)$ & $10.20(5.96)$ & $<0.001$ \\
$\quad$ Anxiety (GAD-7, Range: 0-21) & $5.42(5.26)$ & $4.13(5.51)$ & $4.84(4.94)$ & $6.08(5.16)$ & $7.13(5.42)$ & 0.019 \\
$\quad$ Insomnia (ISI, Range: 0-28) & $5.86(4.97)$ & $5.02(5.18)$ & $5.34(4.53)$ & $6.47(5.04)$ & $7.04(5.38)$ & 0.114 \\
\hline
\end{tabular}

Values are presented as means (SD); ANOVA was used for analyses of group differences. T1, February 2020; T2, January 2021; PHQ-9, Patient Health Questionnaire-9; GAD-7, Generalized Anxiety Disorder-7 item; ISI, Insomnia Severity Index.

males in the prevalence of depression, anxiety, or insomnia.

Differences in depression, anxiety, and insomnia between T1 and T2 among college students were also evaluated as a function of different years in college (grades), with results presented in Figure 2. Students in Grade 4 had a greater prevalence for depression at $\mathrm{T} 2$ than at $\mathrm{T} 1$ $(p=0.03)$, but no statistically significant differences were obtained for anxiety and insomnia. In contrast, no significant differences in the prevalence of depression, anxiety, and insomnia between T1 and T2 were observed in students within Grades 1-3. Results from our ANOVA revealed the differences in depression and anxiety levels among college students within the different grades. We found that at T1, significant differences in levels of depression $(p=0.007)$ and anxiety $(p=0.001)$ were present among college students in different grades (Table 3). Specifically, Grade 4 students showed greater levels of depression $(p=0.005)$ and anxiety $(p=0.008)$ than Grade 1 students (online suppl. Table 2). At T2, there were overall significant differences in depression $(p<0.001)$ and anxiety $(p=0.019)$, with Grade 4 students demonstrating greater levels of depression than Grade 1 students $(p=$ $0.004)$. No statistically significant differences were obtained among the 4 grades for insomnia, at either the T1 or T2 periods.

\section{Discussion}

In the present study, a prospective investigation via an online survey was conducted to assess the mental health status of college students at universities in Jinan City during the peak and reopening stages of the COVID-19 epi- demic. Our findings provide the first evidence indicating that a greater prevalence and more severe depression and anxiety were experienced during the reopening stage as compared with that of the peak stage of this epidemic. These results were quite unexpected and contrary to our hypothesis. Among these students, those in Grade 4 demonstrated the maximal levels of depression prevalence at both peak and reopening stages. These findings not only identify some of the mental health problems induced by the COVID-19 epidemic among these college students but also provide the foundation for the development of effective coping strategies in the planning and management of their mental health problems.

At 3 months after the initial lockdown of Wuhan on January 23, 2020, the COVID-19 epidemic in China was essentially brought under control. As a result, toward the end of April 2020, "normal" activities in China gradually reopened [25], and college students returned to campus life, albeit while wearing masks and maintaining social distancing to prevent further COVID-19 outbreaks [26]. According to recent reports, the pressure of academic delays resulting from online classes as experienced during the peak stage was more prominent during the reopening stage of this epidemic $[27,28]$. Contributing to the concerns and fears associated with the ongoing COVID-19 epidemic was the uncertainty and variety of negative information regarding the outbreak's resurgence [29]. Given these uncertainties and academic pressure, we wondered whether the mental health impact of the COVID-19 epidemic on college students during the reopening stage might be less than that of the peak stage, an issue that has yet to be addressed. Somewhat surprisingly, our findings revealed that the prevalence and severity of depression and anxiety among college students during the reopening 
stage were greater than that experienced during the peak stage of this epidemic.

Results from previous studies have shown that in the absence of timely psychological interventions, people exposed to epidemics, including students, experience persistent psychological problems and diminished social functioning even at several months post-epidemic exposure $[12,30,31]$. In college students, moderate or severe anxiety and depression inevitably impair their academic success and future personal interactions [10]. Therefore, the self-report results in our study have important clinical implications. One approach to address these self-rating reports of moderate to severe anxiety or depression in college students is to encourage more comprehensive face-to-face interviews for their clinical diagnosis and/or psychological intervention [32, 33]. Moreover, as college students subjected to social distancing may develop psychological problems over time $[12,34]$, it becomes necessary to implement a long-term monitoring plan, which includes continuous screening, timely detection, and resolution of possible psychological problems, from the peak to reopening stages, and even to post-epidemic periods. Here, we report that although the peak of the COVID-19 epidemic in China had passed, the psychological problems of these college students remained severe. These findings emphasize the necessity for long-term care and psychological interventions to alleviate the mental health problems encountered by these college students.

Our results further revealed that female college students experienced a greater prevalence of depression than males during the peak stage of the COVID-19 epidemic, suggesting that females might be more sensitive to the initial COVID-19 outbreak. This finding is consistent with previous studies indicating that women are more vulnerable to stress- and fear-based disorders, such as anxiety, depression and post-traumatic stress disorder [35-37]. Interestingly, although females experienced a greater prevalence of depression at the initial peak stage, males and females both showed greater levels of depression in the reopening stage. Therefore, females may require additional attention during the initial stages of an epidemic, while similar coping strategies may be effective for males and females during the reopening stage.

An additional finding of interest was the significantly greater levels of depression and anxiety of Grade 4 versus Grade 1 students during the peak and reopening stages of the COVID-19 epidemic. Moreover, Grade 4 students showed an overall greater prevalence of depression during the reopening stage. Given this global recessionary environment, the greater prevalence of depression among
Grade 4 college students might involve pressures related to employment $[38,39]$. It has been reported that the economic recession associated with the peak stage of the COVID-19 outbreak in China generated additional employment pressures upon the public, including Grade 4 college students [9]. Accordingly, during the reopening stage, Grade 4 students faced more difficult challenges as associated with seeking employment opportunities, which were further restricted due to the worldwide COVID-19 epidemic. In this regard, social support networks such as providing more information on available jobs or actively promoting economic development would serve as an important strategy to reduce the employment pressure and prevent the development of psychological problems in these Grade 4 college students. Our research findings provide a bases and clarification of key points for social education departments to develop strategies for improvement of the mental health of college students.

The present study has some limitations. First, the psychological assessments employed were based on an online survey with little information and on self-report scales, which may introduce a bias with regard to participants' choices. As additional factors, such as allostatic load, lifestyle habits, psychological well-being, or household income levels, may contribute to depression and anxiety prevalence and levels among college students [9], the use of clinical interviews is encouraged in future studies to generate a more comprehensive assessment of psychological problems. Second, the number of students who participated in the survey was relatively small which may, in part, be due to some students voluntarily opting out of participation in this study. Finally, as it was not possible to understand the features of those outside the survey, we cannot certify that the participants of this study were representative of all the college students at universities within Jinan.

\section{Conclusion}

In brief, the mental status of college students at universities within Jinan during the peak and reopening stages of the COVID-19 epidemic were assessed. Greater levels of prevalence and a more severe degree of depression and anxiety were observed during the reopening versus peak stage of this epidemic, with Grade 4 college students being most seriously affected. These results reveal that college students require positive approaches, including adequate social support and long-term targeted psychological interventions. In specific, the more serious mental 
health problems of Grade 4 students warrant programs directed toward increasing their employment opportunities along with recovery plans targeted at improving their mental health.

\section{Acknowledgements}

The authors would like to thank all participants for their time and excellent cooperation.

\section{Statement of Ethics}

Informed consent was obtained from all subjects involved in the study. The research was approved by the Medical Ethics Committee of the Second Hospital of Shandong University (No. KYLL2020 (LW)-062). All procedures contributing to this study comply with the ethical standards of the relevant national and institutional committees on human experimentation and with the Helsinki Declaration of 1975, as revised in 2008.

\section{Conflict of Interest Statement}

The authors have no conflicts of interest to declare.

\section{Funding Sources}

H.W. was supported by grants from the National Natural Science Foundation of China (81771862), and Shandong University humanities and social science major project (No. 19RWZD14). The funding organizations had no role in the design and conduct of the study; collection, management, analysis, and interpretation of the data; preparation, review, or approval of the manuscript; and decision to submit the manuscript for publication.

\section{Author Contributions}

P.W., X.Z., and H.W. conception and design; X.Z., W.M., Y.G., H.Y., L.W., X.Z., Q.L., J.B., and P.W. conduction; X.Z. and L.L. statistical analysis; X.Z., W.M., Y.G., L.W., Q.L., J.B., and X.Z. administrative, technical, or material support; X.Z. drafting of the manuscript; and P.W. and H.W. critical revision of the manuscript for important intellectual content. All authors read and approved the final paper.

\section{Data Availability Statement}

The data that support the findings of this study are not publicly available due to their containing information that could compromise the privacy of research participants but are available from the corresponding author upon reasonable request.

\section{References}

1 Sohrabi C, Alsafi Z, O’Neill N, Khan M, Kerwan A, Al-Jabir A, et al. World Health Organization declares global emergency: a review of the 2019 novel coronavirus (COVID-19). Int J Surg. 2020;76:71-6.

2 Li Q, Guan X, Wu P, Wang X, Zhou L, Tong $\mathrm{Y}$, et al. Early transmission dynamics in $\mathrm{Wu}-$ han, China, of novel coronavirus-infected pneumonia. N Engl J Med. 2020;382(13): 1199-207.

3 World Health Organization. WHO coronavirus disease (COVID-19) dashboard internet: World Health Organization; 2020 [cited 2020 Dec 5]. Available from: https://covid19.who. int/.

4 Wiersinga WJ, Rhodes A, Cheng AC, Peacock SJ, Prescott HC. Pathophysiology, transmission, diagnosis, and treatment of coronavirus disease 2019 (COVID-19): a review. JAMA. 2020;324(8):782-93.

5 Wang C, Pan R, Wan X, Tan Y, Xu L, Ho CS, et al. Immediate psychological responses and associated factors during the initial stage of the 2019 coronavirus disease (COVID-19) epidemic among the general population in China. Int J Environ Res Public Health. 2020; 17(5): 1729 .

6 Lai J, Ma S, Wang Y, Cai Z, Hu J, Wei N, et al. Factors associated with mental health outcomes among health care workers exposed to coronavirus disease 2019. JAMA Netw Open. 2020;3(3):e203976.

7 Cao W, Fang Z, Hou G, Han M, Xu X, Dong J, et al. The psychological impact of the COVID-19 epidemic on college students in China. Psychiatry Res. 2020;287:112934.

8 Liu J, Zhu Q, Fan W, Makamure J, Zheng C, Wang J. Online mental health survey in a medical college in China during the COVID-19 outbreak. Front Psychiatry. 2020;11: 459.

9 Peng M, Wang L, Xue Q, Yin L, Zhu BH, Wang K, et al. Post-COVID-19 epidemic: allostatic load among medical and nonmedical workers in China. Psychother Psychosom. 2021;90(2):127-36.

10 Kivlighan DM, Schreier BA, Gates C, Hong JE, Corkery JM, Anderson CL, et al. The role of mental health counseling in college students' academic success: an interrupted time series analysis. J Couns Psychol. 2020.

11 Richardson M, Abraham C, Bond R. Psychological correlates of university students' academic performance: a systematic review and meta-analysis. Psychol Bull. 2012;138(2): 353-87.

12 Wu P, Fang Y, Guan Z, Fan B, Kong J, Yao Z, et al. The psychological impact of the SARS epidemic on hospital employees in China: exposure, risk perception, and altruistic accep- tance of risk. Can J Psychiatry. 2009;54(5): 302-11.

13 Jeong H, Yim HW, Song YJ, Ki M, Min JA, Cho J, et al. Mental health status of people isolated due to Middle East Respiratory Syndrome. Epidemiol Health. 2016;38:e2016048.

14 Shandong. There are 10 universities announced back to school time. Shandong: Dazhong Daily; 2020 [cited 2020 May 19]. Available from: http://sd.offcn.com/html/ 2020/05/354773.html.

15 Tang W, Hu T, Hu B, Jin C, Wang G, Xie C, et al. Prevalence and correlates of PTSD and depressive symptoms one month after the outbreak of the COVID-19 epidemic in a sample of home-quarantined Chinese university students. J Affect Disord. 2020;274:1-7.

16 Mizumoto K, Kagaya K, Chowell G. Early epidemiological assessment of the transmission potential and virulence of coronavirus disease 2019 (COVID-19) in Wuhan City, China, January-February, 2020. BMC Med. 2020; 18(1):217.

17 Headline News April 30, 2020, 10 PM Internet: China Plus; 2020 [cited 2020 Apr 30]. Available from: http://chinaplus.cri.cn/podcast/detail/2/52048.

18 Kroenke K, Spitzer RL, Williams JB. The PHQ9: validity of a brief depression severity measure. J Gen Intern Med. 2001;16(9):606-13. 
19 Kendler KS. The genealogy of major depression: symptoms and signs of melancholia from 1880 to 1900. Mol Psychiatry. 2017; 22(11):1539-53.

$20 \mathrm{Wu}$ Y, Levis B, Riehm KE, Saadat N, Levis AW, Azar M, et al. Equivalency of the diagnostic accuracy of the PHQ-8 and PHQ-9: a systematic review and individual participant data meta-analysis. Psychol Med. 2020;50(8): 1368-80.

21 Wang W, Bian Q, Zhao Y, Li X, Wang W, Du $J$, et al. Reliability and validity of the Chinese version of the patient health questionnaire (PHQ-9) in the general population. Gen Hosp Psychiatry. 2014;36(5):539-44.

22 Lowe B, Decker O, Muller S, Brahler E, Schellberg D, Herzog W, et al. Validation and standardization of the generalized anxiety disorder screener (GAD-7) in the general population. Med Care. 2008;46(3):266-74.

23 Plummer F, Manea L, Trepel D, McMillan D. Screening for anxiety disorders with the GAD-7 and GAD-2: a systematic review and diagnostic metaanalysis. Gen Hosp Psychiatry. 2016;39:24-31.

24 Bastien CH, Vallières A, Morin CM. Validation of the Insomnia Severity Index as an outcome measure for insomnia research. Sleep Med. 2001;2(4):297-307.

25 Huynh DL, Ngau TH, Nguyen NH, Tran GB, Nguyen CT. Potential therapeutic and pharmacological effects of Wogonin: an updated review. Mol Biol Rep. 2020;47(12):9779-89.
26 National Health Commission. The National Health Commission further improves the ability of pre-hospital medical emergency response under the normalization of the prevention and control of COVID-19 epidemic Internet: General Office of the National Health Commission; 2020 [cited 2020 Jul 14]. Available from: http://www.gov.cn/zhengce/ zhengceku/2020-07/14/content_5526762. htm.

27 Biswas S, Biswas A. Anxiety level among students of different college and universities in India during lock down in connection to the COVID-19 pandemic. Z Gesundh Wiss. 2021:1-7.

28 Dangal G, Bajracharya LS. COVID-19 in pregnancy: pregnant women might be at greater risk for severe COVID-19. Kathmandu Univ Med J. 2020;18(2):1-2.

29 Merow C, Urban MC. Seasonality and uncertainty in global COVID-19 growth rates. Proc Natl Acad Sci U S A. 2020;117(44):27456-64.

30 Main A, Zhou Q, Ma Y, Luecken LJ, Liu X. Relations of SARS-related stressors and coping to Chinese college students' psychological adjustment during the 2003 Beijing SARS epidemic. J Couns Psychol. 2011;58(3):410-23.

31 McAlonan GM, Lee AM, Cheung V, Cheung C, Tsang KW, Sham PC, et al. Immediate and sustained psychological impact of an emerging infectious disease outbreak on health care workers. Can J Psychiatry. 2007;52(4):241-7.
32 Lamond JE, Joseph RD, Proverbs DG. An exploration of factors affecting the long term psychological impact and deterioration of mental health in flooded households. Environ Res. 2015;140:325-34.

33 Adams RE, Boscarino JA, Galea S. Social and psychological resources and health outcomes after the World Trade Center disaster. Soc Sci Med. 2006;62(1):176-88.

34 Liu X, Kakade M, Fuller CJ, Fan B, Fang Y, Kong J, et al. Depression after exposure to stressful events: lessons learned from the severe acute respiratory syndrome epidemic. Compr Psychiatry. 2012;53(1):15-23.

35 Maeng LY, Milad MR. Sex differences in anxiety disorders: interactions between fear, stress, and gonadal hormones. Horm Behav. 2015;76:106-17.

36 Faravelli C, Alessandra Scarpato M, Castellini G, Lo Sauro C. Gender differences in depression and anxiety: the role of age. Psychiatry Res. 2013;210(3):1301-3.

37 Labaka A, Goñi-Balentziaga O, Lebeña A, Pérez-Tejada J. Biological sex differences in depression: a systematic review. Biol Res Nurs. 2018;20(4):383-92.

38 Kecojevic A, Basch CH, Sullivan M, Davi NK. The impact of the COVID-19 epidemic on mental health of undergraduate students in New Jersey, cross-sectional study. PLoS One. 2020;15(9):e0239696.

39 Kapasia N, Paul P, Roy A, Saha J, Zaveri A, Mallick R, et al. Impact of lockdown on learning status of undergraduate and postgraduate students during COVID-19 pandemic in West Bengal, India. Child Youth Serv Rev. 2020;116:105194. 